\title{
Correlation Between Axial Length of Eyeball And Peripapillary Rnfl Thickness Measured By Oct in Myopes.
}

\author{
Dr. Jitendra Kumar ${ }^{1}$,Dr. Shweta Dwivedi ${ }^{2}$,Dr. Arun Kumar Pathak ${ }^{3}$, \\ Dr. Amit Verma ${ }^{4}$ \\ 1,2,3,4 (Department Of Ophthalmology,M.L.B.Medica Lcollege/Bundelkhand University,India)
}

\begin{abstract}
This study was conducted to determine the correlation between axial length of eye ball and peripapillary RNFL thickness measured by OCT in myopes. The study was carried out on patients attending Outpatient Department Of Ophthalmology at M.L.B Medical College, Jhansi. Myopic subjects >18 years of age without other ocular pathology were invited to participate. Subjects for the study were selected based on strict inclusion and exclusion criteria as indicated. All subjects underwent a thorough ophthalmic examination (FMR) to confirm the lack of ocular pathology other than refractive error .

Refractometry was performed in both eyes and converted to spherical equivalents. The more myopic eye was selected as the study eye.. The study eye was examined for axial length biometry. Slit lamp biomicroscopy was done to look for the appearance of optic nerve (including vertical and horizontal disk diameter, cup-to-disk ratios, and the presence of disk tilt and/or peripapillary atrophy) and and documented by Stratus OCT ( including vertical and horizontal disk diameter, disk area, and vertical and horizontal cup-to-disk ratio). Significant and strong association was found between axial length and RNFL thickness( $p$ value $=0.002$ ). Significant and less strong association was found between spherical number and RNFL thickness ( $p$ value $=0.006)$. The stronger correlation with axial length could furthermore indicate that globe elongation (with geometrically concomitant thinning of the globe wall) is an important determinant of thin RNFL.

Thin RNFL measurements in myopes could represent a predisposing factor for the

future development of glaucoma, which could partly explain why glaucoma is more prevalent

among myopes
\end{abstract}

Keywords: RNFL, Axial lengh, OCT, Myopes, Spherical number.

\section{Introduction}

Myopia is the most common ocular abnormality worldwide. Retinal changes in persons with high myopia include peripapillary atrophy, peripheral lattice degeneration, tilting of the optic disc, posterior staphyloma and breaks in Bruch's membrane[1]. Although retinal nerve fiber layer thinning is indicative of glaucomatous damage, it remains uncertain whether retinal nerve fiber layer thickness would vary with the refractive status of the eye)[2-3] It is therefore important to investigate whether any correlation exists between retinal nerve fiber layer thickness and axial length in myopia. The aim of this study is very important because it measures retinal nerve fiber layer thickness changes with the increase in axial length as in myopia. The decreasing retinal nerve fiber layer thickness is a major risk factor to develop glaucoma which is a second most common cause of blindness worldwide[4]

Glaucoma is associated with increased cupping of the optic disk and thinning of the retinal nerve fiber layer (RNFL). Although glaucoma is commonly diagnosed by the appearance of the optic disk, the optic disk is quite variable in appearance within the normal population.Therefore, RNFL analysis, using instruments such as Stratus optical coherence tomography (OCT), has been proposed as a new way to evaluate for glaucoma[5].

Myopia has been reported as a risk factor for glaucoma, and myopic fundus changes maycomplicate glaucoma diagnosis and management[6]. Conflicting data exist regarding theinfluence of myopia on peripapillary retinal nerve fiber layer (RNFL) thickness[7]. Some showno association[8-11] whereas Budenz et al.[12] and Leung et al.[13] found significant correlations. Thepurpose of this study is to assess whether longer axial length and increasing axial myopia arecorrelated with thinner RNFL measurements.

\section{Materials And Methods}

A Prospective study was conducted at the OPD of Department Of Ophthalmology at M.L.B Medical College, Jhansi. Myopic subjects $>18$ years of age without other ocular pathology were invited to participate . Subjects for the study were selected based on strict inclusion and exclusion criteria as indicated.

\section{Inclusion Criteria}

Myopic subjects $>18$ years of age without other ocular pathology were invited to participate. 


\section{Exclusion criteria}

Any Morbidity like diabetes, cataract Use of photosensitizing agents. History of ocular surgery All subjects underwent a thorough ophthalmic examination (FMR) to confirm the lack of ocular pathology other than refractive error. All subjects had intraocular pressure of $18 \mathrm{~mm} \mathrm{Hg}$ or less in both eyes. Refractometry was performed in both eyes and converted to spherical equivalents. The more myopic eye was selected as the study eye.. The study eye was examined for axial length biometry. The appearance of the optic nerve was documented by slit lamp biomicroscopy (including vertical and horizontal disk diameter, cup-to-disk ratios, and the presence of disk tilt and/or peripapillary atrophy) and Stratus OCT (including vertical and horizontal disk diameter, disk area, and vertical and horizontal cup-to-disk ratio).

Disk tilt was defined as 30 to 60 degrees of tilt along the nasal-temporal axis, resulting in a prominent nasal rim and temporally-displaced optic cup. Atrophy was defined as a crescent of discoloration beginning at the disk margin and extending $>0.2 \mathrm{~mm}$ but $<0.8 \mathrm{~mm}$ from the disk margin. The study eye underwent an OCT examination of the peripapillary RNFL using the Fast RNFL algorithm, provided with the Stratus OCT instrument. The algorithm was run four times, producing twelve RNFL scans per subject (each run of the algorithm gives three scans). The overall mean RNFL thickness and mean thickness for each quadrant (superior,

inferior, temporal, and nasal) was calculated by averaging the results from all 12 scans.

Linear regression was used to determine the correlation between RNFL thickness and continuous variables (axial length, sphere, and age). T-tests and analysis of variance were usedto determine significant associations between RNFL thickness and non-continuous variables (demographic and optic disk characteristics). Significance was set at $\mathrm{p}<0.05$.

\section{Results}

Thirty- six subjects were taken for the study. One subject was excluded due to history of strabismus surgery. Of the 35 included subjects, 15 were female and 20 male. The mean age was $34 \pm 8$ years (range 2354) with average axial length of $25.70 \mathrm{~mm}$ (range 22.50 to 27.83). Seventy five percent had axial length of $>25$ $\mathrm{mm}$. Mean spherical equivalent was -5.50 (range -1.50 to -11.50 ). Mean cup-to-disk ratio by fundoscopy was 0.38, but measured slightly higher when measured by the Fast Optic Disk algorithm on the OCT- machine (horizontally 0.56 , vertically 0.57 ). Thirteen subjects (37\%) had temporal peripapillary atrophy and Seven $(20 \%)$ had temporal disk tilt. No atrophy or tilt was found at the inferior, nasal, or superior disk margins. Twenty two subjects $(62.8 \%)$ had neither atrophy nor tilt. No significant associations were found between RNFL thickness and age $(\mathrm{p}=0.25)$, gender, cup-to-disk ratio, tilt, or peripapillary atrophy..A significant, strong association was found between axial length and RNFL thickness (Fig.1a). A significant but less strong relationship was found between spherical equivalent and RNFL thickness (Fig. 1b).

RNFL thickness decreased with higher axial length (Overall $\mathrm{R}=-0.70, \mathrm{p}<0.001$, Superior $\mathrm{R}=-0.60$, $\mathrm{p}=0.001$, Inferior $\mathrm{R}=-0.60, \mathrm{P}=0.001$ )(fig. $2 \mathrm{a} ; 3 \mathrm{a}$ ) higher spherical equivalent (Overall $\mathrm{R}=-0.52, \mathrm{p}=0.005$, Superior $\mathrm{R}=-0.41, \mathrm{p}=0.03$, Inferior $\mathrm{R}=-0.45, \mathrm{p}=0.02)$ (Fig. 2b ; 3b)

Overall RNFL thickness decreased 6 microns for every $1 \mathrm{~mm}$ of axial length, and 2.5 microns for every 1 Diopter sphere. Nasal and temporal RNFL thickness showed no significant associations with myopia (Fig. 4 \&5). Representative OCT- scans are shown in (Fig. 6). Note that thin RNFL in myopic subjects occurred preferentially at the superior and inferior poles.Fourteen subjects $(40 \%)$ had RNFL thickness scans that could be classified as "outside normal limits," defined as one or more reproducible yellow quadrants on the OCT RNFL scan (Fig. 6). Two of these subjects had a reproducible red quadrant. When comparing low versus high myopes (using sphere of -5.00 Diopters as a cutoff), 50\% (7/14) of high myopes had abnormal quadrants, versus $15 \%$ $(2 / 13)$ of low myopes $(\mathrm{p}=0.002)$. Similar numbers were found when axial length of $25.50 \mathrm{~mm}$ was used as the cutoff: $53 \%$ ( $8 / 15$ high myopes) versus $8 \%$ (1/12 low myopes), $\mathrm{p}=0.001$. The mean overall RNFL thickness was found to be yellow(outside normal limits) in six subjects, all of whom had axial length greater than $25.50 \mathrm{~mm}$ $(\chi 2, p=0.017)$. None of the subjects had a red overall RNFL thickness reading.Overall, $19(54 \%)$ of these 35 "normal" myopic subjects had an RNFL thickness reading "outside normal limits," as defined by a reproducible yellow clock-hour, quadrant, or mean measurement.

\section{Discussion}

Budenz et al.[12]. evaluated the determinants of RNFL thickness in the normative database of Stratus OCT. In their study, they noted a significant decrease in RNFL thickness with increasing axial length, equal to 2.2 microns per $\mathrm{mm}$. Their mean axial length was $23.8 \mathrm{~mm}$, with mean refractive error of -0.54 Diopters and mean age of 47 years. One misconception regarding the normative database for Stratus OCT is that extremes of refractive error were excluded. On the contrary, there were no exclusionary criteria based on refractive error for the normative database and the range of spherical equivalents in the normative data was +6.75 to -11.75 . 
We hypothesized that moderate to high myopes might have a more pronounced decrease in RNFL thickness that could place them outside the normal range, as defined by the OCT . To test this hypothesis, we chose a significantly more myopic sample, enriched for spherical equivalents greater than 4 diopters $(70 \%$ of our subjects), who were also younger and more homogenous for age This could theoretically help avoid labeling myopic individuals as glaucoma suspects based on their Stratus OCT RNFL scans. This is crucial because myopes often have visual field defects that simulate glaucoma, and difficult to assess optic nerves[8].

Several prior studies did not find a significant association between myopia and RNFL thickness $[9,10,14,15]$. These studies may have been limited by the poorer resolution of earlier generation OCT and confocal laser devices, and thus lower sensitivity. Using the current generation Stratus OCT, Salchow et al[11]. found that RNFL thickness increased with increasing hyperopia (1.7 microns per diopter) in children (91\% Hispanic, mean age 10 years, range 4-17). Only five children with $>4.00$ diopters of myopia were assessed, which was too small for analysis. Using an older generation of OCT technology to study macular thickness, Lim et al.[16] found that myopes had a significantly thicker fovea but thinner parafovea. They suggested that axial myopia may result in a flattening, and perhaps "thinning out" of the retinal layers, including the nerve fiber layer; however, they found that overall macular thickness outside the fovea was not associated with axial length.

Our present study, as well as work by Leung et al.[13] found a lack of correlation between age and RNFL thickness in myopic subjects. This may reflect the relatively young population and narrow age range used in both studies (average age of 34 years and 36 years). Budenz et al.[12] found a small but significant correlation with age ( 2 microns of RNFL thinning per decade) in a population with age equally distributed between 20 and 70 years (mean age 47 years, range 18-85). Likely, when factors such as age are held relatively constant (our age range was confined to three decades), the other factors (e.g. refractive error) become more prominent.

The fact that thin RNFL correlated more strongly with axial length than with spherical equivalent is not surprising, since population variations in the refractive power of the cornea and lens could affect refractometry(but likely not the axial length). The stronger correlation with axial length could furthermore indicate that globe elongation (with geometrically concomitant thinning of the globe wall) is an important determinant of thin RNFL.

The decrease in RNFL thickness with axial length (6 microns $/ \mathrm{mm}$ ) was higher in our study population than previously published for other populations (Budenz et al.[12] 2 microns $/ \mathrm{mm}$, Leung et al.[13] $2.75 \mathrm{microns} / \mathrm{mm}$, Salchow et al.[11] 1.7 microns $/ \mathrm{mm}$ ). Furthermore, our study is the first to demonstrate that the overall decrease in RNFL thickness measurements are due in particular to thin RNFL at the superior and inferior poles of the optic disk (Fig. 2a ; 3a). Importantly, thin polar RNFL could be misattributed to glaucomatous change if one does not account somehow for the effect of axial length, such as by adjusting current OCT nomograms. Leung et al.[13] found that Chinese myopic subjects had thin nasal RNFL, a finding not borne out in our population. Thin RNFL could be related to an elongation and thinning of the retina and sclera, which would spread the nerve fibers over a larger surface area.[13] It could also represent, in part, an actual decrease in nerve fiber number, although this does not appear to be the case based on histologic analysis.[17]

Severe peripapillary atrophy or tilt may affect RNFL measurements by OCT.[18,19] However, only mild temporal tilt $(n=7)$ or atrophy $(n=13)$ were present in our subjects, and neither one correlated with either overall or temporal RNFL thickness. Thus thin RNFL measurements in our moderate to high myopes appeared to be related to axial lengthening rather than to small amounts of atrophy around the optic nerve head. However, due to the subjective nature of our methodology to measure tilt and atrophy, these cannot be completely ruled out as factors by this study.

Other technologies, such as scanning laser polarimetry, have yielded conflicting results regarding myopia and RNFL measurements.[20-23] Choplin and colleagues did not find a relationship between nerve fiber parameters and axial length or refractive error using scanning laser polarimetry with fixed corneal compensation in 235 normal subjects.[20] However, unlike the current study, this study excluded subjects with 5 diopters or more of spherical refractive error. The relatively normal refractive errors and axial lengths in these subjects, as well as the narrow range, may explain the lack of a relationship in this study. Decreases in RNFL seen with scanning laser polarimetry, such as following keratorefractive surgery for myopia, have been largely attributed to artifacts, such as alterations of corneal birefringence rather than to true thinning of the RNFL.[2123] The current OCT findings might similarly have resulted from an artifact of the measurement instrument. OCT technology relies on the time-delay of backscattered light coming from the eye, compared to a reference beam. It is possible that lower RNFL measurements are recorded in myopes due to differences in reflectivity or back-scatter in certain longer eyes, as opposed to actual differences in RNFL thickness or nerve fiber count. In addition, histologic data do not support the notion of nerve fiber "loss," as fiber counts have been reported to be equal across normal eyes of different sizes.[17] 
Finally, thin RNFL measurements in myopes could represent a predisposing factor for the future development of glaucoma, which could partly explain why glaucoma is more prevalent among myopes in some studies.[4-7] A long-term, longitudinal study of our subjects, to determine for example if their OCT "defects" are progressive, would of course be necessary to discern such a predisposition. Doshi et al. followed sixteen young male Chinese myopes with arcuate visual field defects, simulating glaucoma but likely related to optic nerve tilt, for seven years without treatment, and none were found to progress.[19] Our findings could indicate that a milder version of this phenomenon, with OCT RNFL defects but normal visual fields, may be present in our young moderate myopes.

In addition, the homogenous, young nature of our subjects provided enhanced sensitivity by controlling for other factors that could have affected RNFL measurements. Weaknesses included our relatively small sample size, although the magnitude and strength of the findings indicate that only a small sample is needed to be sufficiently powered.

The small age range chosen meant that the study would not be powered to detect a small effect of age on RNFL thickness. In contrast, the even distribution of refractive errors over a large 10 diopter range meant that the study would be uniquely powered to detect the effect of myopia on RNFL. The current study suggests that myopes undergoing RNFL analysis by Stratus OCT should ideally be compared to a normative control group that is matched for refractive error and/or axial length. Until then, we recommend careful interpretation of RNFL data on moderate to highly myopic individuals (particularly those with axial length $>25 \mathrm{~mm}$ ) when applying the currently available OCT devices.

\section{CONCLUSION}

In conclusion, our study provides a reliable correlation values between axial length and retinal nerve fiber layer thickness in myopic eyes by optical coherence tomography. This can serve as a useful guideline in diagnosis, management and research in glaucoma in myopic eyes.

\section{REFERENCES}

[1]. Masood S, Hussain Z, Ahmad A, Tanveer ZH, Ahmad I. Incidence of myopia in school going children in Rahim Yar Khan Professional Med J. 2007; 14: 422-5.

[2]. Alam H, Siddiqui I, Jafri SI, Khan AS, Ahmed SI, Jafar M. Prevelance of refractive error in school children of Karachi. J Pak Med Assoc. 2008; 58: 322-5.

[3]. Budenz DL, Anderson DR, Varma R. Determinants of normal retinal nerve fiber layer thickness measured by Stratus OCT. Ophthalmology. 2007; 114: 1046-52.

[4]. In focus: Glaucoma is second leading cause of blindness globally. Bull World Health Organ. 2004; $82: 887$.

[5]. Wong TY, Klein BE, Klein R, et al. Refractive errors, intraocular pressure, and glaucoma in a white population. Ophthalmology 2003;110:211-217. [PubMed: 12511368]

[6]. Mitchell P, Hourihan F, Sandbach J, Wang JJ. The relationship between glaucoma and myopia: the Blue Mountains Eye Study. Ophthalmology 1999;106:2010-2015. [PubMed: 10519600]

[7]. Xu L, Wang Y, Wang S, et al. High myopia and glaucoma susceptibility the Beijing Eye Study. Ophthalmology 2007;114:216-220. [PubMed: 17123613]

[8]. Jonas JB, Dichtl A. Optic disc morphology in myopic primary open-angle glaucoma. Graefes Arch Clin Exp Ophthalmol 1997;235:627-633. [PubMed: 9349946]

[9]. Garcia-Valenzuela E, Anderson NG, Pons M, Iezzi R. Retinal thickness by OCT in subjects with emmetropia, hyperopia, and myopia. Invest Ophthalmol Vis Sci 2002;43E-Abstract 2574

[10]. Hoh ST, Lim MC, Seah SK, et al. Peripapillary retinal nerve fiber layer thickness variations with myopia. Ophthalmology 2006;113:773-777. [PubMed: 16650672]

[11]. Salchow DJ, Oleynikov YS, Chiang MF, et al. Retinal nerve fiber layer thickness in normal children measured with optical coherence tomography. Ophthalmology 2006;113:786-791. [PubMed: 16650674]

[12]. Budenz DL, Anderson DR, Varma R, et al. Determinants of normal retinal nerve fiber layer thickness measured by Stratus OCT. Ophthalmology 2007;114:1046-1052. [PubMed: 17210181]

[13]. Leung CK, Mohamed S, Leung KS, et al. Retinal nerve fiber layer measurements in myopia: An optical coherence tomography study. Invest Ophthalmol Vis Sci 2006;47:5171-5176. [PubMed: 17122099]

[14]. Kashiwagi K, Tamura M, Abe K, et al. The influence of age, gender, refractive error, and optic disc size on the optic disc configuration in Japanese normal eyes. Acta Ophthalmol Scand 2000;78:200-203. [PubMed: 10794257]

[15]. Melo GB, Libera RD, Barbosa AS, et al. Comparison of optic disk and retinal nerve fiber layer thickness in nonglaucomatous and glaucomatous patients with high myopia. Am J Ophthalmol 2006;142:858-860. [PubMed: 17056370]

[16]. Lim MC, Hoh ST, Foster PJ, Lim TH, Chew SJ, Seah SK, Aung T. Use of optical coherence

[17]. tomography to assess variations in macular retinal thickness in myopia. Invest Ophthalmol Vis Sci 2005;46:974-978. [PubMed: 15728555]

[18]. Jonas JB, Schmidt AM, Muller-Bergh JA, et al. Human optic nerve fiber count and optic disc size. Invest Ophthalmol Vis Sci 1992;33:2012-2018. [PubMed: 1582806]

[19]. Lai E, Wollstein G, Price LL, et al. Optical coherence tomography disc assessment in optic nerveswith peripapillary atrophy. Ophthalmic Surg Lasers Imaging 2003;34:498-504. [PubMed:14620759]

[20]. Doshi A, Kreidl KO, Lombardi L, et al. Nonprogressive glaucomatous cupping and visual field abnormalities in young Chinese males. Ophthalmology 2007;114:472-479. [PubMed: 17123617]

[21]. Choplin NT, Lundy DC, Dreher A. Differentiating patients with glaucoma from glaucoma suspects and normal subjects by nerve fiber layer assessment with scanning laser polarimetry. Ophthalmology 1998;105:2068-76. [PubMed: 9818608] 
[22]. Choplin NT, Schallhorn SC. The effect of excimer laser photorefractive keratectomy for myopia on nerve fiber layer thickness measurements as determined by scanning laser polarimetry. Ophthalmology 1999;106:1019-1023. [PubMed: 10328407]

[23]. Ozdek SC, Onol M, Gürelik G, Hasanreisoglu B. Scanning laser polarimetry in normal subjects and patients with myopia. Br J Ophthalmology 2000;84:264-267.

[24]. Choplin NT, Schallhorn SC, Sinai M, et al. Retinal nerve fiber layer measurements do not change after LASIK for high myopia as measured by scanning laser polarimetry with custom compensation. Ophthalmology 2005;112:92-97. [PubMed: 15629826]

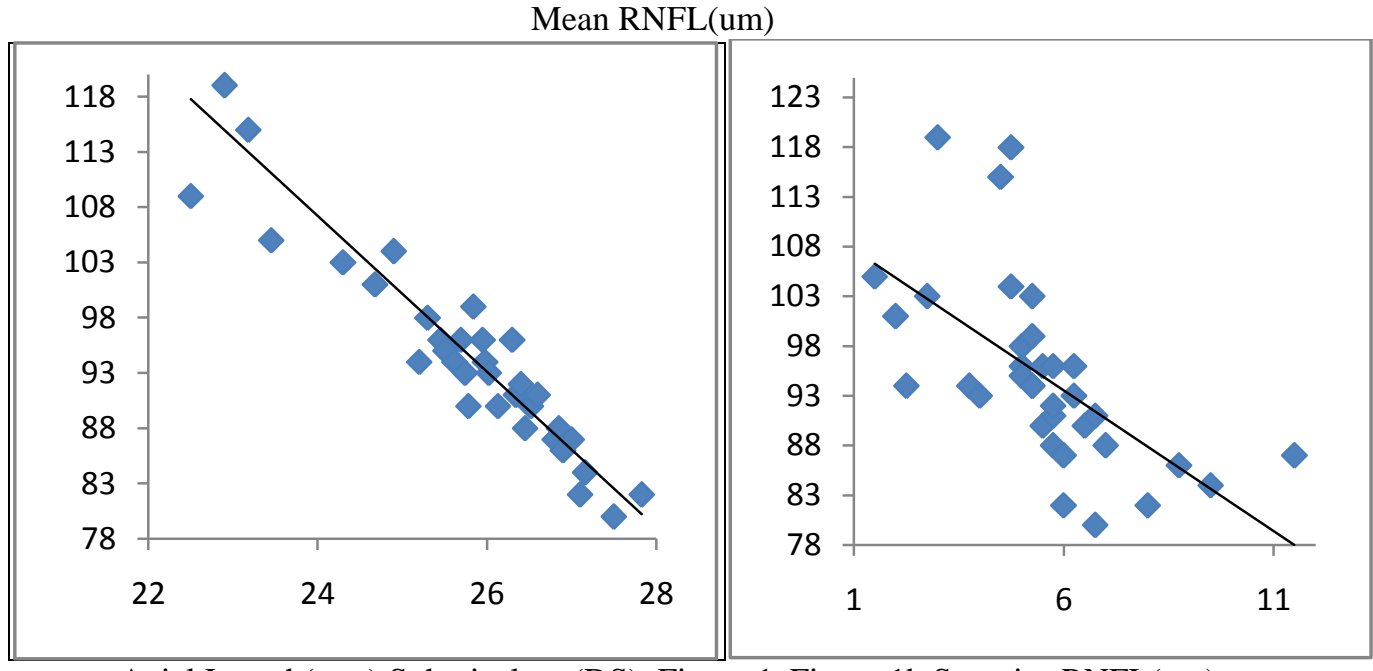

Axial Length(mm) Spherical no.(DS) Figure 1aFigure 1b Superior RNFL(um)

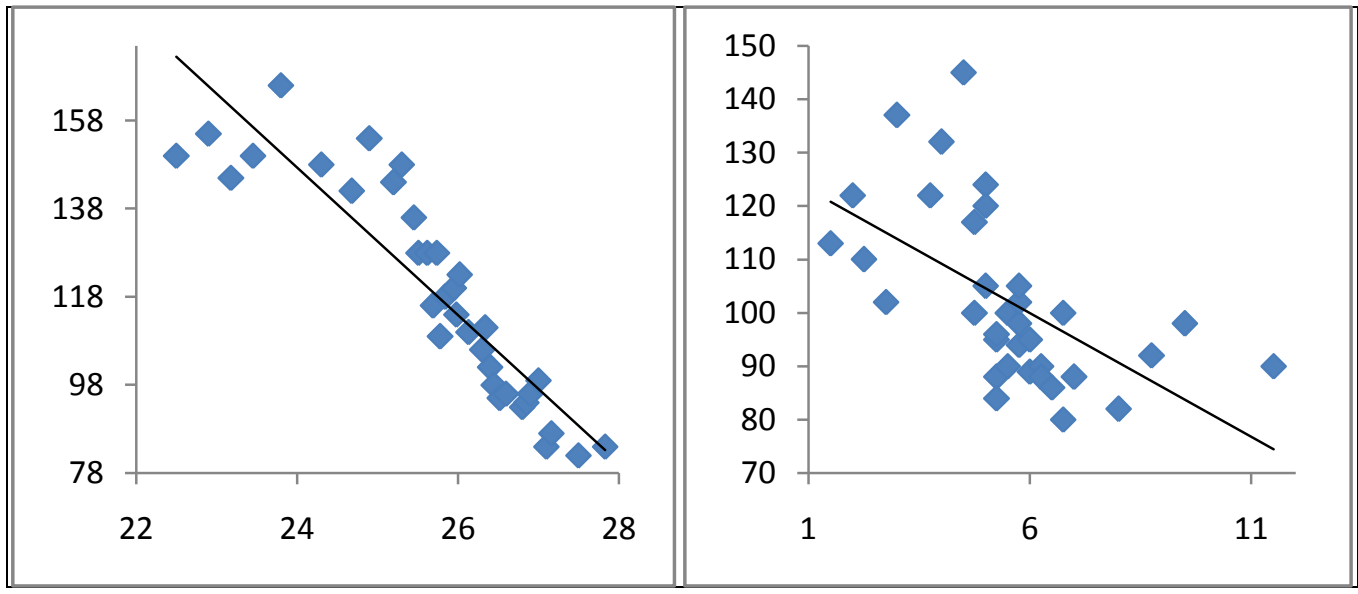

Axial Length(mm) Spherical no.(DS)Figure 2a,Figure 2b Inferior RNFL(um)

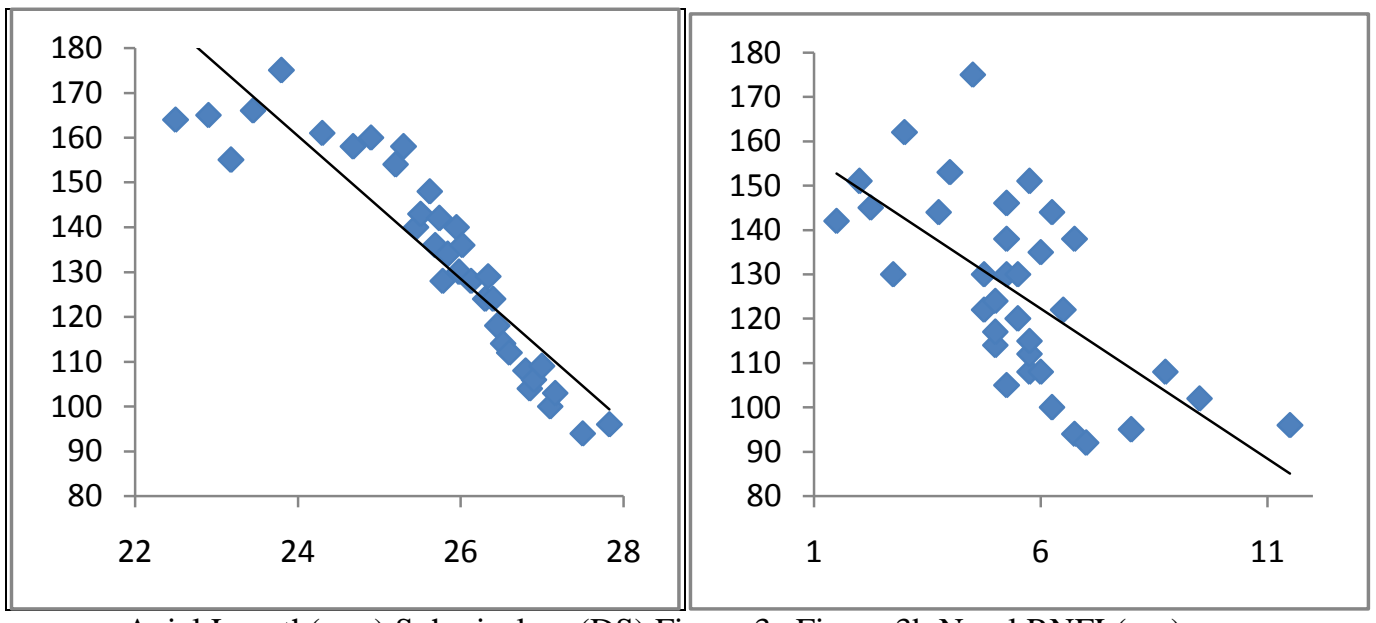

Axial Length(mm) Spherical no.(DS) Figure 3a Figure 3b Nasal RNFL(um) 


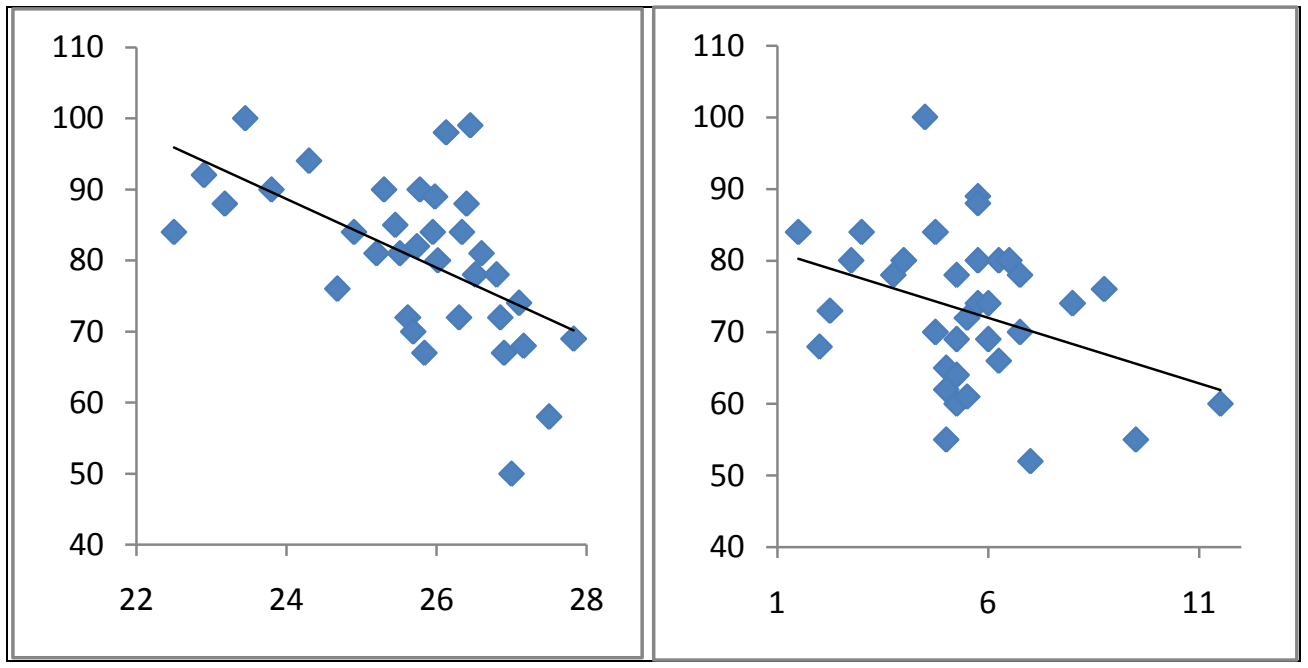

Axial Length(mm) Spherical no.(DS) Figure 4aFigure 4b Temporal RNFL(um)

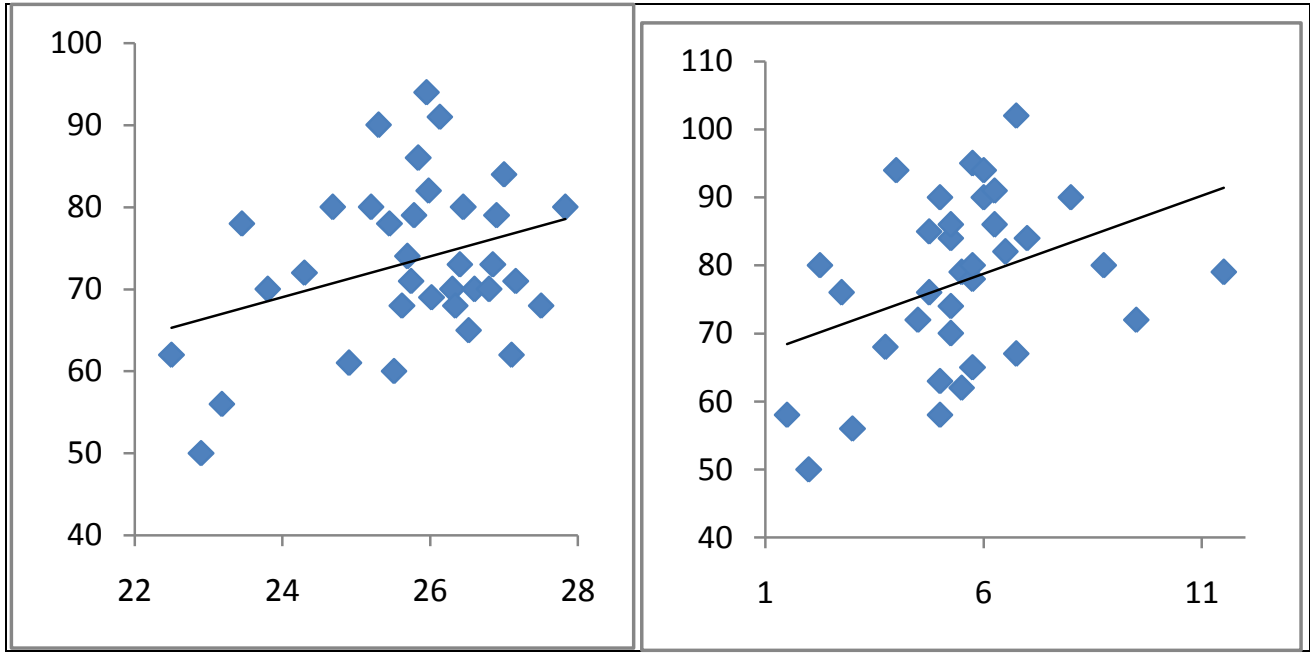

Axial Length(mm) Spherical no.(DS) Figure 5a Figure 5b
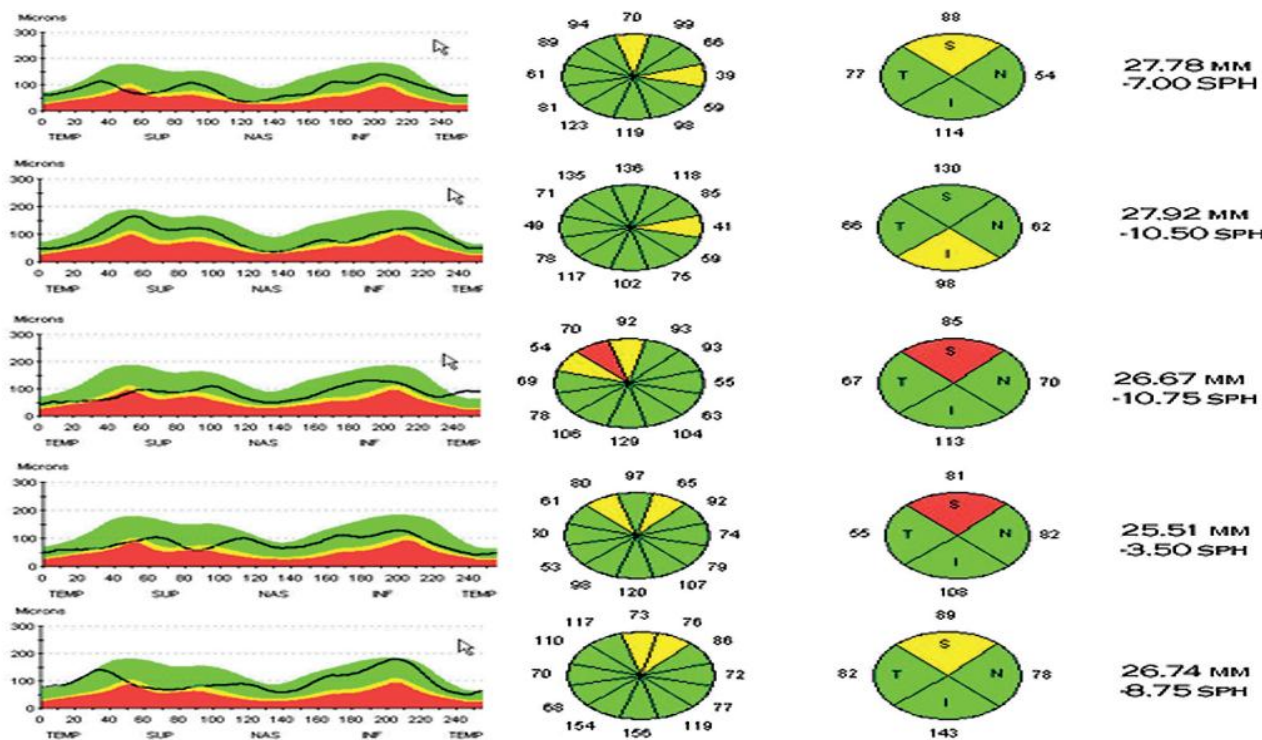

$26.74 \mathrm{~mm}$

Figure 6.

OCT showing Retinal Nerve Fiber Layer (RNFL) scans of myopic subjects. It is to be noted that thinning of RNFL occur preferentially at the superior and inferior poles of the optic disk. 